\title{
Emerging clinical applications of computed tomography
}

This article was published in the following Dove Press journal:

Medical Devices: Evidence and Research

5 June 2015

Number of times this article has been viewed

\section{Carlo Liguori ${ }^{1}$ \\ Giulia Frauenfelder ${ }^{2}$ \\ Carlo Massaroni ${ }^{3}$ \\ Paola Saccomandi ${ }^{3}$ \\ Francesco Giurazza ${ }^{4}$ \\ Francesca Pitocco 4 \\ Riccardo Marano ${ }^{5}$ \\ Emiliano Schena ${ }^{3}$}

'Radiology Unit, AORN A Cardarelli, ${ }^{2}$ Radiology Unit, AOU Federico II, Naples, ${ }^{3}$ Measurement and Biomedical Instrumentation Unit, ${ }^{4}$ Radiology Unit, Università Campus Bio-Medico di Roma, ${ }^{5}$ Department of Radiological Sciences, Institute of Radiology, Catholic University of Rome, A Gemelli University Hospital, Rome, Italy
Correspondence: Emiliano Schena Measurement and Biomedical Instrumentation Unit, Università Campus Bio-Medico di Roma, Via Álvaro del Portillo 21, 00128 Rome, Italy Tel +3906 2254I 9650 Email e.schena@unicampus.it
Abstract: X-ray computed tomography $(\mathrm{CT})$ has recently been experiencing remarkable growth as a result of technological advances and new clinical applications. This paper reviews the essential physics of X-ray CT and its major components. Also reviewed are recent promising applications of CT, ie, CT-guided procedures, CT-based thermometry, photon-counting technology, hybrid PET-CT, use of ultrafast-high pitch scanners, and potential use of dual-energy CT for material differentiations. These promising solutions and a better knowledge of their potentialities should allow CT to be used in a safe and effective manner in several clinical applications.

Keywords: computed tomography, X-ray, thermometry, dual-energy, ultrafast scanner, guidance, photon-counting technology

\section{Introduction}

Many diagnostic imaging techniques, including ultrasound imaging, magnetic resonance (MR) and computed tomography (CT), have gained widespread acceptance in several fields of medicine. In spite of the concern related to the patient dose, CT imaging has become a "cannot-do-without tool" in many branches of medicine.

The first application of CT dates back to shortly after its invention, when between 1957 and 1963 Cormack applied this technology to improve radiotherapy planning. ${ }^{1}$ The first successful implementation of CT was performed a few years later by Hounsfield, who surprised the entire medical community with his experiments. ${ }^{2}$ From the introduction of CT in the early 1970s, its importance in clinical imaging has exceeded even the most optimistic hopes of researchers. The steadily increasing numbers of examinations based on this technique and the introduction of new procedures performed under CT guidance in clinical practice have promoted research on new applications in this scenario. Nowadays, CT is widely used for both diagnostic and therapeutic procedures.

This article reviews some medical applications of X-ray CT. After a brief description of its major components and some essential physics, particular attention is focused on the characteristics of each type of CT scanner, on the applications of multidetector CT (MDCT) and dual-energy CT (DECT), as well as emerging applications proposed to improve the outcomes of some diagnostic and therapeutic procedures.

\section{History and essential physics History}

The discovery of X-radiation in 1895 by Röntgen, who investigated radiance during electric discharges inside an evacuated glass tube, revolutionized the diagnosis of 
several diseases. ${ }^{3,4}$ After 1895 , X-ray research developed very rapidly, and the first picture of a whole skeleton obtained by X-rays dates back to $1897 .{ }^{3}$ Over the years, the design of X-ray equipment was improved to obtain high quality two-dimensional images of the inside of the human body. ${ }^{3}$ Among others, Edison made a significant contribution to the development of medical imaging techniques, and von Helmholtz investigated the mathematical equations describing properties of X-rays and their penetration through different materials. ${ }^{3}$ Thompson investigated the possibility of obtaining a three-dimensional X-ray image. ${ }^{5}$ These studies led to development of some investigational techniques, such as the ones patented by Baese in 1915 and Bocage in 1922. A further step in the direction of contemporary scanners was the use of gamma radiation to obtain a layered image of tissues, proposed by Kuhn in 1963 . However, the discovery of X-rays was not enough to lead to the advent of CT; in fact, its development was more related to the development of computational techniques. The first commercial fan-beam CT scanner come onto the market in 1973, with an acquisition time of 20 seconds and 30 detectors. In 1979, Cormack and Hounsfield were awarded the Nobel Prize and credited with inventing the modern CT. ${ }^{1,2}$ Since the end of 1970 s, a lot of progress has been made in CT design and manufacture. In comparison with the first scans, contemporary ones can scan in a few hundred milliseconds, and reconstruct an image of $2,048 \times 2,048$ pixels from hundreds of spiral slices.

\section{Essential physics}

$\mathrm{CT}$ is based on the principle that the density of the tissue passed through by the X-ray beam can be measured by calculation of the attenuation coefficient. The X-ray emitter discharges monochromatic photons that produce a high $\mathrm{kV}$ X-ray beam with an average energy of $75 \mathrm{keV}^{6} \mathrm{X}$-rays are generated by physical processes that take place within matter at the atomic level. During generation of X-radiation in X-ray tubes, transition of electrons between the inner shells of of an atom, and the deceleration of charged particles caused by electromagnetic fields within matter occur. ${ }^{7}$ The X-ray spectrum may be recognized as the sum of the energies of both the above-mentioned processes, resulting in discrete characteristic X-rays and continuous X-ray emission, respectively. After X-rays pass through a layer of biological material, the detector measures an attenuated X-ray intensity. ${ }^{6}$ The unattenuated intensity of the $\mathrm{X}$-ray beam, $I_{0}$, is also measured by the $\mathrm{CT}$ scanner.

$\mathrm{X}$-ray monochromatic intensity, $I$, is defined as the amount of photon energy $(N \cdot h v)$ passing through a unit area $(S)$ in unit time $(t)$ :

$$
I=\frac{N \cdot h v}{S \cdot t}
$$

where $h$ is Planck's constant and $v$ is the frequency of the photon of radiation emitted.

The relationship between the $I_{t}$ and $I_{0}$ can be expressed by Lambert-Beer's law: ${ }^{6}$

$$
\ln \left(I_{t} / I_{0}\right)=\mu \cdot x
$$

where $x$ is the thickness of the biological tissue and $\mu$ is the linear attenuation coefficient. CT reconstruction algorithms use Equation (2) in a pre-processing step before image reconstruction to reduce dependence of the CT image on machine-dependent parameters (ie, $I_{t}$ and $I_{0}$ ).

The attenuation of radiation due to interaction with matter is related to some phenomena (ie, photoelectric effect and coherent and incoherent scattering). ${ }^{3}$ The total value of the linear attenuation depends on the substance and tracks linearly with density. ${ }^{8}$

After $\mathrm{CT}$ reconstruction, before storing and displaying, $\mathrm{CT}$ images are normalized to integer values comprising the CT number (also known as the Hounsfield unit), ${ }^{8}$ which defines the degree of attenuation of radiation by various substances. The number $C T(x, y)$ in each image pixel $(x, y)$ is expressed as: ${ }^{6}$

$$
C T(x, y)=1000 \frac{\mu(x, y)-\mu_{\mathrm{H}_{2} \mathrm{O}}}{\mu_{\mathrm{H}_{2} \mathrm{O}}}
$$

where $\mu_{\mathrm{H}_{2} \mathrm{O}}$ is the attenuation coefficient of water. This normalization results in $\mathrm{CT}$ numbers ranging from $-1,000$ to $+3,000$ (eg, $-1,000$ for air and $+3,000$ for dense bone or areas filled with contrast agents). Contrast in CT images mainly derives from the physical properties of tissue that influence incoherent scattering (known as Compton scattering), which depends on tissue electron density, $\rho_{e}=N Z / A$ (where $Z$ and $A$ are the atomic number and atomic mass, respectively). ${ }^{9}$ As a consequence, a tissue containing a relative abundance of hydrogen (eg, fat) is well visualized by CT. The CT number allows accurate diagnosis in some clinical settings, and accurate estimation of, eg, tumor volume and lesion diameter.

The DECT scanner is gaining acceptance in the clinical setting. ${ }^{10}$ The principle underpinning the clinical use of DECT is the dependency of $\mu$ on the X-ray energy. Indeed, each type of material demonstrates a relatively specific change in attenuation between images obtained with different energy spectra, and this difference in attenuation allows a more 
nuanced characterization of the features depicted. With this technique, two image datasets are acquired in the same anatomic location with two different X-ray spectra. Different types of DECT scanners are available, as explained in the section on physics and applications of DECT scanners. Two major advantages of DECT are material decomposition by the almost simultaneous acquisition of two image series with different $\mathrm{kVp}$ values (80 and 140) and elimination of misregistration artifacts.

\section{Key performance parameters and major components of $\mathrm{CT}$ scanners}

The main components of CT scanners are the following: the $\mathrm{X}$-ray genera the system for X-ray detection, the system of collimators and filters, and the system for the reconstruction of images. The patient is positioned within the gantry on a table that moves automatically during the scans. The X-ray tube is responsible for generation of X-rays. These are emitted when matter is hit by charged particles with high kinetic energy $\left(10^{3}-10^{6} \mathrm{eV}\right)$ that are able to knock electrons out of their atomic orbits. Basically, the X-ray tube is composed of a cathode and an anode within a vacuum glass envelope. Electrons from the cathode tungsten filament are accelerated toward the anode, represented by a tungsten target electrode maintained at a positive voltage potential with respect to the cathode. Peak voltage is applied to the cathode, and the tube current, ie, the rate of electron flow from the cathode to the anode, in the order of hundreds of $\mathrm{mA}$, is used to produce X-rays. ${ }^{6}$ The X-rays pass through the body in the gantry and carry the information about the structure of the body to the detectors. The information, represented by radiation intensities, is in the form of a series of projections, measured by the detection system. Usually, the whole detector system rotates synchronously with the X-ray tube around the body. Different detectors are used (ie, xenon detector, solid-state detector, and multiple detector arrays) to detect the projections and convert them into electrical quantities. Such quantities are used by the reconstruction system to form the diagnostic image. Collimators and filters are used to limit unnecessary radiation exposure and to improve the image quality.

A series of X-rays passing through the patient at the same orientation is known as a projection. The main projection geometries used are: parallel beam geometry, where all the rays are parallel to each other; fan beam geometry, in which the rays diverge, looking like a fan; and cone beam geometry, where the beam has a conical shape. Each geometry has a dedicated algorithm for reconstruction of images from the projections. The modern CT scanner incorporates fan beam geometry in the acquisition and reconstruction processes. CT scanner technology has evolved over several generations, and CT scanner technology has evolved over several generations.

The first-generation scanner used a parallel beam system and lateral movement to make a single projection, along with a circular movement about the central opening in the gantry to gather all the projections necessary to reconstruct the image. Only two detectors measured the X-rays transmitted by the patients. The second-generation scanner used a fan beam system and a linear array of 30 detectors, which improved utilization of the X-ray beam by 30 -fold. The slow scanning due to the translational motion of the first-generation and second-generation scanners was overcome by the third-generation scanners, where the X-ray tube and detector array are mechanically joined and rotated together around the patient. The problem of artifact with the third-generation scanner was partially overcome by the fourth-generation scanner. The fifth-generation scanners differ significantly from the others, in that they do not contain moving parts and the conventional $\mathrm{X}$-ray tube is substituted by an arc of tungsten encircling the patient, and an electron beam is steered around the patient, hitting the arc. The sixth-generation scanner is known as the helical CT scanner, because data are acquired while the patient's table is moving. Lastly, the seventh-generation scanner uses a multiple detector array (solid-state detectors), allowing an increased number of X-rays to be detected, and enabling better and more efficient patient imaging.

Many parameters can influence the quality and performance of CT imaging, and can be related to generation of X-rays, phase of acquisition, or steps in image reconstruction.

The performance parameters of CT scanners are indicators of the quality of the system in terms of its physical and technical capabilities, and allow standardized, comparative, and quantitative criteria to be established. The key parameters are: spatial resolution, ie, the minimum area of the image in which changes are detectable; low-contrast resolution, ie, the ability to detect changes in tissue attenuation (typical values are close to $0.4 \%$ ); slice thickness, ie, the nominal thickness of the image cross-section (typical values $0.4 \mathrm{~mm} \div 10 \mathrm{~mm}$ ); pitch, ie, the ratio between displacement of the table and the thickness of the scanned layer for one revolution of the scanner (relevant only for helical CT scanners); and dose, ie, an indicator of the damage that X-rays absorbed by the patient can cause. The CT dose index is the most commonly used dose indicator. The value of the dose absorbed by the patient during a scan is strongly related to a number of parameters 
(eg, tube voltage, current-time product, and thickness and number of slices). The total dose absorbed by the patient should not exceed permissible levels; consequently, efforts of manufacturers of tomographic equipment are directed towards minimizing this dose. ${ }^{3}$

\section{Clinical applications and technical parameters}

With remarkable growth in its applications and use, evolution of MDCT technology has resulted in significant changes in the scanning parameters, hardware, and radiation exposure associated with CT scanning. In comparison with single-detector CT, conventional MDCT allows acquisition of different image slabs from the same dataset by using helical reconstruction, weighting algorithms, and interpolation of adjacent helical datasets.

Salient MDCT characteristics are: a cone-shaped X-ray beam, up to 0.5 seconds of rotation time ( $2 \mathrm{~Hz}$ of frequency), faster scan coverage, larger z-axis coverage/rotation (18/24/32 mm), isotropic voxel possible, greater temporal resolution and less image noise, beam pitch, and reduced radiation dose efficiency. ${ }^{11}$

MDCT has multiple detector rows (each consisting of 500-900 detector elements) along the scanning direction, which create a two-dimensional curved detector array. MDCT acquires more than one slice in a single X-ray tube rotation with thin isotropic voxels. At a beam pitch of $1: 1$, table speed and z-axis coverage from dual-slice CT scanners (20 $\mathrm{mm}$ per second) have steadily increased to $40 \mathrm{~mm}$ per second for four-slice, $80 \mathrm{~mm}$ per second for eight-slice, and $160 \mathrm{~mm}$ per second for 16-slice MDCT scanners. Currently available MDCT scanners have as many as 40 rows of detectors in the z-axis, which comprise more than 30,000 individual detector elements with high efficiency and minimal afterglow. In addition to changes in detector design, evolution of CT technology has resulted in substantial alteration in the image reconstruction techniques used to accommodate changes in image geometry; the filtered back-projection technique of image reconstruction is used in single-slice axial scanners, and interpolated filtered back-projection is used in single-detector CT scanners and four-slice MDCT.

Recent implementation of computer calculation performances has made it possible to introduce into clinical CT scanners a different image reconstruction model known as the iterative reconstruction protocol. This protocol is mainly based on comparison of the acquired CT image with a CT model in order to reduce CT image noise using lower $\mathrm{Kv}$ levels or to improve image quality using the same $\mathrm{kV}$ levels. ${ }^{12}$ Isotropic scanning may be defined as acquisition of images with equal voxel size in three axes: MDCT scanners permit acquisition of thin slices with isotropic voxel size.

Images obtained using conventional MDCT provide useful but only limited material-specific information, because the representation of structures on images depends solely on the linear attenuation coefficient of each of the constituent materials and is independent of the material density and mass attenuation coefficient.

The introduction of DECT technology made possible new diagnostic scenarios: two datasets $(80 \mathrm{kVp}$ and $140 \mathrm{kVp})$ are loaded on the workstation, and virtual non-contrast, iodine map, and mixed (with adjustable blending of $80 \mathrm{kVp}$ and 140 $\mathrm{kVp}$ data) images can be obtained. ${ }^{10}$

\section{Single-energy CT applications of multidetector scanners}

MDCT offers several improvements when performing CT angiography. Because scans are faster, less intravenous contrast is necessary, resulting in cost-savings and a reduced contrast load to the patient's kidneys. Moreover, the scan distance is increased so that the entire volume (ie, chest, abdomen, and pelvis) can be scanned in one acquisition; this is a definite advantage when scanning the aorta, because only one acquisition is necessary. Thinner collimation $(0.5-1.0 \mathrm{~mm})$ improves visualization of small vessels $(<1 \mathrm{~mm})$, which is helpful when staging malignancies, evaluating organ donors and patients with suspected pulmonary embolism, and visualizing the mesenteric vessels.

The introduction of MDCT resulted in advances in musculoskeletal imaging. Multiplanar reconstruction can be created in any plane, maintaining high resolution (the joint needs to be scanned only in one plane, preferably oblique to the joint surface). CT colonography involves volumetric CT imaging after colonic cleansing combined with sophisticated processing software to create three-dimensional endoluminal views. ${ }^{12}$

MDCT also allows scanning of the heart during a single breath hold, eliminating artifact caused by respiratory motion; moreover, it allows noninvasive characterization of cardiac arrhythmias using an integrative approach that combines electrical and structural data (electrocardiography and MDCT). ${ }^{13}$

\section{Physics and applications of DECT scanners}

Physics

The basic principle of DECT or spectral imaging is to obtain two datasets with different $\mathrm{kVp}$ values (usually 80 and 140) 
from the same anatomic region. This allows performing material decomposition thanks to the attenuation differences at different energy levels. X-ray attenuation of materials in the diagnostic energy range varies according to the specific organ composition and X-ray beam energy. Consequently, different substances show a different CT number at different energies. The increase in photon energy results in a small decrease in CT values for materials with a low atomic number while it causes a rapid decrease in Hounsfield unit values for materials with a high atomic number. ${ }^{14}$

At low $\mathrm{kVp}$, the photoelectric effect predominates for elements with a high atomic number, such as calcium and iodine. With a reduction in $\mathrm{kVp}$, the incidence of photoelectric interaction increases. In contrast with single-spectrum imaging, DECT is sensitive to the chemical composition. This means that DECT is capable of differentiating materials with different atomic numbers, despite similar attenuation coefficients, because the data are acquired using two different spectra. Consequently, materials with almost similar coefficient attenuation values at different energy levels can be distinguished by DECT. ${ }^{15}$

\section{Post-processing}

There are two approaches to extracting dual-energy information from projection data. A straightforward method is to subtract equivalent projections and apply filtered backprojection to reconstruct the difference as spectral information. Another way is to consider the CT number of voxels in standard CT images, and then to use post-processing algorithms to extract specific spectral information from the difference between the corresponding voxels. Currently, the most commonly used approach is the latter, with the image reconstruction system providing low-kVp and high-kVp images and a series of weighted average images. Three main types of algorithms are in use: the first one optimizes images; the second one identifies or differentiates certain materials; and the third one quantifies a substance in the dataset. The output of the first algorithm consists of altered gray-level CT images, whereas the output of the latter two algorithms usually color-code substances, either several substances in different colors or the quantity of one substance on a color palette.

Optimization algorithms are monoenergetic images in which the CT number for each voxel is extrapolated to a certain energy from the two density values at the acquired photon energies, and nonlinear blending algorithms. Differentiation algorithms define a slope between the density values obtained by scanning the material at the two spectra, and differentiate materials on the basis of the photoelectric effect. Quantification algorithms use a three-material decomposition, quantifying one of three materials. ${ }^{16}$

Research in the technical development of DECT led to four different scanner models, sharing the same technological concept but being different in terms of acquisition structure and produced by different manufacturers as follows:

- Dual-source and dual-energy scanner (Definition Flash and Force; Siemens Healthcare, Forchheim, Germany) which obtains high-energy scans at $120 / 140 \mathrm{kVp}$ and lowenergy scans simultaneously at $80 / 100 \mathrm{kVp}$ (limited in temporal registration and field of view in large patients). This technology is consistently helped by high-end (Stellar) detectors with miniaturized electronic components able to generate ultra-thin slices with a very high spatial resolution. Moreover, a selective photon shield panel blocks low-energy photons out of the high-energy $\mathrm{X}$-ray spectrum and so prevents unnecessary exposure.

- Single-source and dual-energy scanner with fast $\mathrm{kV}$ switching (Revolution CT; GE Healthcare, Milwaukee, WI, USA). As a result of Gemstone detector technology, a fast $(0.25 \mathrm{msec})$ temporal sampling of $80 \mathrm{kVp}$ and $140 \mathrm{kVp}$ switching during a single gantry rotation is achievable in order to generate high-energy and lowenergy X-ray spectra (they are limited in individual modification of the high-energy and low-energy X-ray beams because of the single X-ray source, not yet possible on commercially available scanners; moreover, the spectral overlap increases).

- Single-source and dual-energy scanner with dual detector layers (Brilliance CT; Philips Healthcare, Andover, MA, USA) with a modified detector array (two scintillation layers arranged one atop the other) to receive separate high-energy and low-energy image data streams from a single X-ray source (not yet available for routine clinical use).

- Single-source and dual-energy scanner with faster gantry rotation and able to generate high-energy and low-energy $\mathrm{X}$-ray spectra on same anatomic position in course of two separate rotations (Aquilion ONE; Toshiba, Nasu, Japan).

\section{Applications of DECT scanners}

Two types of clinically useful quantitative information can be generated by DECT, ie, the physical densities of known basis material pairs (eg, iodine and water) and the effective atomic number of a substance located in a selected region of interest. The physical density of a material can be measured directly from material density images. 
Using DECT, noncontrast (unenhanced) images can be avoided by using the dual-energy mode for different clinical applications: iodine can be removed from the image and a virtual noncontrast (water) image can be acquired. The major advantage of $80 \mathrm{kVp}$ images compared with $140 \mathrm{kVp}$ images is a higher image contrast. Typically, a combination of $80 / 140 \mathrm{kVp}$ is used for DECT, but $100 / 140 \mathrm{kVp}$ is preferred for some applications. ${ }^{10}$ With material characterization algorithms, iodine can be differentiated from other tissues on a contrast-enhanced DECT scan. The dual-energy software then subtracts iodine from all regions of the image, generating a virtual unenhanced image. On this image, enhancing lesions can be distinguished from calcification and other highattenuation lesions, without having the patient undergo scanning before administration of contrast. ${ }^{17}$ Moreover, DECT can generate iodine distribution images or maps on which the calculated iodine distribution on an image is color-coded and superimposed on the virtual unenhanced images.

Potential applications of DECT according to anatomic regions usage can be grouped as follows.

\section{Head-neck}

Excellent anatomic detail is preserved and lesions can be easily delineated from their surroundings because of superimposition of a color map on the original CT images. ${ }^{12}$ For instance, invasion of laryngeal cartilage by squamous cell carcinoma can be challenging to assess on single-energy CT images because uncalcified or unossified cartilage has attenuation similar to that of the enhancing tumor. A DECT protocol using $100 \mathrm{kVp}$ and $140 \mathrm{kVp}$ is really useful for evaluation of potential cartilage involvement. ${ }^{18}$

\section{Lung}

DECT may improve detection of pulmonary embolism in comparison with conventional CT and may assist in evaluation of lung perfusion (Figure 1A and B). Regional distribution of ventilation can also be assessed by administerinh xenon to the patient as a contrast material instead of iodine. DECT can also evaluate pulmonary nodule characteristics by using virtual nonenhanced images. ${ }^{19}$

\section{Abdomen \\ Kidney}

DECT may improve the characterization of smaller indeterminate renal lesions: a hyperattenuating renal lesion on a conventional single-phase (venous-phase) CT scan would be an indeterminate finding that necessitates further work-up. Water and iodine material density images generated from a single-phase DECT dataset can be used to differentiate a small simple cyst from a hemorrhagic cyst or small renal mass: a simple cyst appears dark on both, while a hemorrhagic cyst appears bright on the water display and dark on the iodine display; a solid mass appears isodense to adjacent solid renal parenchyma on water material density images, but its iodine content makes it look brighter than either a simple or a complicated cyst on iodine material density images. ${ }^{20,21}$ Water material density images may also be useful to identify calculi at excretory phase CT because they are
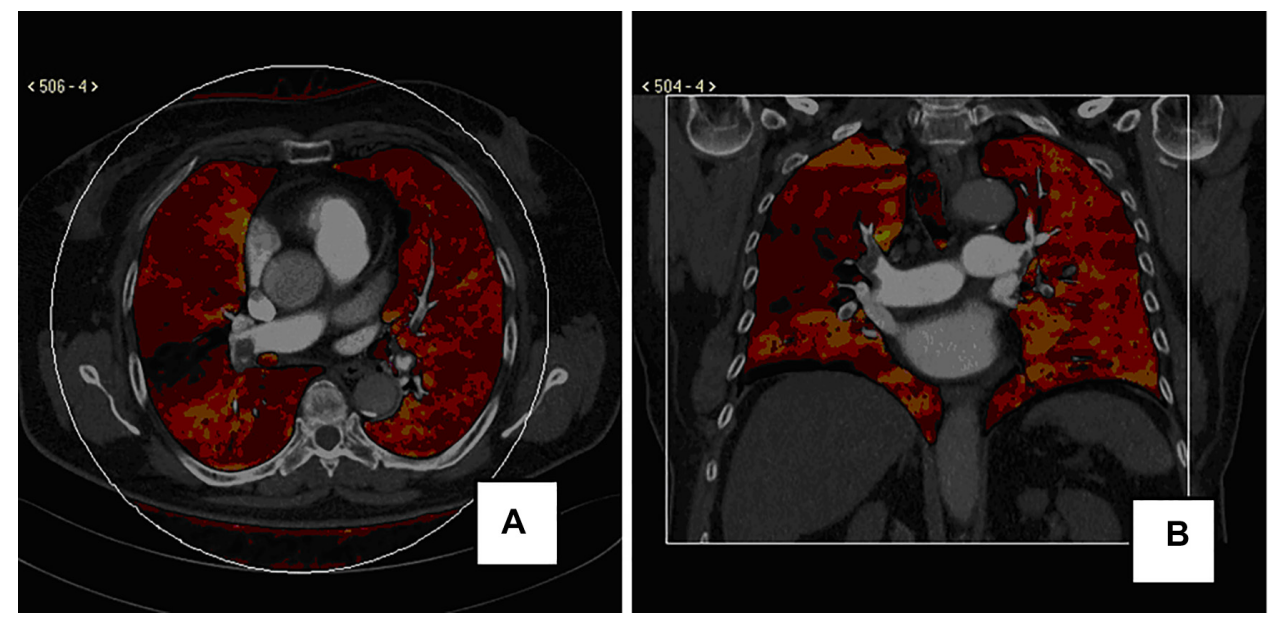

Figure I (A) Dual-energy CT axial image in a patient with chest pain and shortness of breath. Color-coded perfusion map demonstrates a wedge-shaped perfusion defect in the right lower lobe, coupled with an opacification defect of the proximal interlobar pulmonary artery in the weighted average CT mediastinal image. Small emboli can be seen in the left arterial pulmonary branches without parenchyma perfusion defects. (B) Dual-energy CT coronal multiplanar reconstruction in a patient with dyspnea. Color-coded perfusion map shows patchy area of reduced perfusion in the upper and lower right lobe; a weighted average CT image in the mediastinum demonstrates an incomplete obstruction of the upper and lower pulmonary artery. Minimal thrombotic obstructions in the left main pulmonary artery without perfusion alterations in the left lung can be appreciated.

Abbreviation: CT, computed tomography. 
reconstructed by extracting all iodine-containing pixels from the renal collecting system and ureters. Another emerging application of DECT is renal stone characterization, distinguishing between those with and without uric acid. ${ }^{22,23}$

\section{Liver}

A single-phase scan performed with DECT can allow differentiation of small masses from cysts by use of water and iodine material density displays. Simple cysts in the liver appear dark in comparison with adjacent hepatic parenchyma on water material density images and show no iodine-induced enhancement on iodine material density images. Conversely, solid metastases are relatively less well defined and appear isodense or nearly isodense to solid hepatic parenchyma on water material density images and show enhancement on iodine material density images. ${ }^{24} \mathrm{DECT}$ can detect liver iron load: in recent years, several noninvasive methods have been pursued for quantification of iron concentration in the liver. Of these, MR imaging using gradient multiecho sequences has proven to be the gold standard method. Fisher et al performed an ex vivo study to evaluate the accuracy of DECT in quantifying liver iron concentration, showing the added value of this technique. ${ }^{25}$ The three-material decomposition algorithm used in that study was specifically designed to quantify iron; similar to iodine, iron shows an energy-dependent change in $\mathrm{CT}$ attenuation. Using this algorithm, the authors showed a high accuracy of DECT in quantification of liver iron concentration even in the presence of fat. ${ }^{25}$

\section{Pancreas}

DECT may also be helpful for differentiating enhancing pancreatic parenchyma from acute hemorrhage without needing an additional unenhanced acquisition. In addition, DECT may have a role in delineating organ perfusion in the setting of severe acute pancreatitis, helping clarify which regions show reduced perfusion and which regions show necrosis, aiding patient care and the prognosis. ${ }^{24}$

\section{Vascular system}

Imaging evaluation after endovascular repair of abdominal aortic aneurysm generally involves a multiphasic CT protocol. DECT, with a single-phase examination, has the potential to be as accurate as conventional dual-phase CT, with a global reduction in the radiation dose..$^{26,27}$

\section{Heart}

Clinical applications of cardiac DECT have been described for dual-energy perfusion with or without the adenosine stress test, viability imaging, and cardiac iron detection. ${ }^{28-30}$ Ruzsics et al described the combination of dual-energy cardiac perfusion and coronary $\mathrm{CT}$ to diagnose coronary artery stenosis and myocardial ischemia. ${ }^{28}$ Zhang et al investigated the detection of acute myocardial infarction in a canine model using DECT, showing a sensitivity and specificity of $92 \%$ and $80 \%$, respectively. ${ }^{31}$ Bauer et al investigated late enhancement DECT in order to identify areas of chronic myocardial infarction and viability, comparing their results (eg, a sensitivity of $77 \%$ and specificity of $97 \%$ ) with $3 \mathrm{~T}$ MR imaging data. ${ }^{32}$ Several studies have also shown the role of DECT in characterization of plaques, in calcific plaque removal from coronary arteries, and in evaluation of coronary stents in in vitro and ex vivo settings. ${ }^{33,34}$ Cardiac iron load can be detected by DECT, and this technique could be useful for patients who cannot undergo cardiac MR imaging due to claustrophobia or other contraindications ${ }^{35}$ El-Sayed et al showed the power of DECT for evaluating iron overload in the clinical setting with accuracy similar to that of the MR T2* imaging technique. ${ }^{36}$ DECT, in fact, allows evaluation of iron overload without being affected by energy-dependent CT attenuations, having the potential to be an alternative modality for assessing tissue iron overload. The low radiation dose of the imaging protocols on new scanners, very short scan times, ability to identify large iron concentrations, and high resolution make DECT a promising tool for evaluating myocardial iron overload.

\section{Dose exposure issue and indications for usage of ultra-high pitch scanners}

Historically, initial dual-energy imaging approaches came at the expense of a doubled radiation dose and sometimes the need for a second injection of contrast medium. Only since 2006, with the introduction of dual-source CT, have multiple energy image acquisition methods achieved clinical significance and widespread application for diagnostic purposes. Since then, various strategies for acquiring DECT data have been proposed for use with recent generations of advanced MDCT systems: simultaneously applying two X-ray tubes and two corresponding detectors at different $\mathrm{kVp}$ and tube current settings with dual-source $\mathrm{CT}$, rapid $\mathrm{kVp}$ switching based on single-source $\mathrm{CT}$, compartmentalization of detected $\mathrm{X}$-ray photons into energy bins by double-layer detectors of a single-source $\mathrm{CT}$ scanner operating at constant $\mathrm{kVp}$ and tube current settings, synchronized double rotations at different $\mathrm{kVp}$ levels with volume $\mathrm{CT}$, and counting of photons and integrating the $\mathrm{X}$-ray energy flux..$^{37,38}$ There is strong evidence that DECT imaging with dual-source CT technology is not 
associated with increased radiation dose levels. In recent years, several studies have evaluated the use of DECT and shown substantial clinical benefits. However, this exciting advance coincided with a general awareness regarding the use of ionizing radiation for medical imaging and concern about the cumulative radiation dose. Nowadays, the literature suggests that there is no increase in radiation exposure when DECT protocols, based on dual-source CT technology, are used instead of single-energy techniques; Moreover, a potential decrease in radiation dose via virtual unenhanced DECT has been shown in several studies. ${ }^{21,39}$

Several studies have underlined the prognostic value of MDCT in patients suspected to have coronary artery disease, still having concern about radiation exposure and related lifetime cancer risk, especially in younger patients. ${ }^{40,41}$ The helical cardiac CT scan with the retrospective gating technique is still the most commonly used acquisition mode in cardiac CT angiography, using low pitch values (0.2-0.4) and retrospectively adapting the reconstruction time windows, but may still result in a relatively high effective radiation dose $(10-18 \mathrm{mSv}){ }^{42}$

Prospective triggering has recently entered routine clinical practice: suing this acquisition mode, the tube is switched on only during a certain phase of the cardiac cycle, which is prospectively determined from the electrocardiogram, using the R-wave as the scan trigger. This scan technique works best for a regular heart rate below 70 beats per minute, lowering the radiation dose to values around $2-4 \mathrm{mSv}^{43}$

Highly reduced temporal resolutions only became possible after introduction of DECT, obtaining the required data for image generation in just over a quarter gantry rotation, scanning the entire heart in a fraction of a second with so-called high-pitch protocols; these scanners achieve pitch values up to 3.4 without image reconstruction gaps. ${ }^{44}{ }^{46} \mathrm{~A}$ high-pitch fast protocol allows imaging of the entire coronary circulation in a single heart beat ( 0.27 seconds for $12 \mathrm{~cm}$ coverage): in that way, image quality is more independent of heart rate and phase of the cardiac cycle. ${ }^{46}$ The second-generation 128-slice DECT uses a pitch value of 3.2 in the high-pitch spiral scan mode, in which prospective electrocardiography-triggered spiral data acquisition is completed within a single cardiac cycle for sufficiently short scan ranges and sufficiently long inter-beat (R-R) intervals; in this way, the chest is covered in $700 \mathrm{msec}$, obtaining information about the heart and entire chest, opening up new and interesting scenarios in terms of comprehensive cardiothoracic imaging. The second detector array is used to fill in the sampling gaps, and cross-sectional images are obtained from the acquired data with a temporal resolution of $75 \mathrm{msec}$ and an offset of approximately 0.65 msec. ${ }^{47}$ Radiation exposure is also minimized in high-pitch DECT, obtaining a radiation dose $<1 \mathrm{mSv}$ and reducing the dose by $>50 \%$ compared with systems from previous generations. ${ }^{36,48,49}$ Sommer et al showed that patients with regular and low heart rates ( $<65$ beat $/ \mathrm{min})$, and scanned with high-pitch protocols, obtained high image quality and a low number of nonevaluable segments. Their study showed that, for the high-pitch protocol, there is a potential reduction of patient dose of $89.8 \%$, compared with the retrospective gating, and of $61.2 \%$, compared with prospective triggering: hence, the high-pitch protocol showed a great benefit in terms of lifetime attributable to cancer risk of cardiac $\mathrm{CT}^{43}$

\section{Future research}

Two of the most promising emerging solutions in terms of the potential clinical impact of CT are: CT thermometry, which aims at controlling the amount of tissue damaged during hyperthermal procedures; and CT-guided procedures, which allow accurate positioning of either a needle or an applicator for diagnostic and therapeutic purposes. In this section, the basis for and the most significant challenges of these promising solutions are described. Moreover, there is a section devoted to description of other emerging applications, including integration of positron emission tomography (PET) with CT scanners, X-ray/MR systems, and phase-contrast $\mathrm{X}$-ray imaging $(\mathrm{PCI})$.

\section{$\mathrm{CT}$ thermometry}

Much recent research effort has been devoted to introduction of noninvasive techniques for monitoring of temperature during hyperthermal treatment. ${ }^{49}$ Knowledge of tissue temperature may be particularly beneficial, allowing the operator to visualize the running procedure and to be notified in real time about its outcome. ${ }^{50}$ Noninvasive techniques are recommended in this field. ${ }^{51,52}$

Although thermometry based on MR imaging is the most widely employed technique for monitoring the effects of thermal procedures, several research groups have now focused their attention on assessment of the feasibility of CT thermometry in hyperthermal treatments. ${ }^{53,54}$

This technique is based on the dependency of the attenuation coefficient, and consequently the CT number, on temperature. This dependency can be explained by the phenomenon of thermal expansion of the tissue. This relationship can be linearized over a large temperature range, although some authors have proposed quadratic or cubic models. ${ }^{55,56}$

Bydder and Kreel initially investigated the variation in CT number of water with temperature during the 1970s, and in the 
early 1980s Fallone et al assessed the feasibility of this technique to monitor the temperature of biological tissue. ${ }^{57,58}$ After these studies, CT thermometry fell into obscurity due to the poor stability and precision of CT scanners. ${ }^{59}$ However, in the last decade, the high stability of the modern CT scanners have fostered application of CT thermometry. Its feasibility has been assessed during different types of hyperthermal procedures in ex vivo models, on phantoms, and in a preliminary study involving an in vivo pig model. ${ }^{60-62}$ Although CT-based thermometry is in its infancy, recent improvement in the performance of CT scanners and the increasing interest in monitoring of temperature during thermal procedures are helping to increase the number of studies focusing on this technique.

\section{CT-guided procedures}

CT also plays an essential role in the field of interventional radiology. CT-guided interventions consist of a wide set of procedures, divided in diagnostic and therapeutic, which are part of extravascular interventional radiology.

\section{Diagnostic procedures}

The first reported case of use of CT to guide a biopsy dates back to $1975 .{ }^{63}$ The operator handles a needle that needs to be advanced into the tissues up to the target. Different needles can be used, and the choice depends on the site and dimensions of the lesion. CT is considered by far the most accurate method to guide tissue sampling. Almost all suspicious lesions in the human body, except for those in the central nervous system, can be histologically characterized by CT-guided biopsies. Further, biopsy is also required for reassessment of patients with cancer, in order to plan personalized therapies. ${ }^{64}$

CT allows optimal visualization of the needle into the tissues in two or more consecutive slides, guiding the advances of the needle placement. In addition, $\mathrm{CT}$ allows visualization of the needle on multiple planes as a result of post-processing reconstructions (Figure 2). However, the importance of CT lies in immediate post-procedural control; a typical example is CT of the thorax performed after a lung biopsy to detect possible pneumothorax.

\section{Therapeutic procedures}

CT is extensively used to guide aspiration and drainage of collection and abscesses in the abdomen or pleural cavity; the intervention consists of arriving at the target with a needle, using the same technique described previously for biopsies, positioning a guide wire, and then advancing tube of drainage that presents multiple holes on the distal portion.

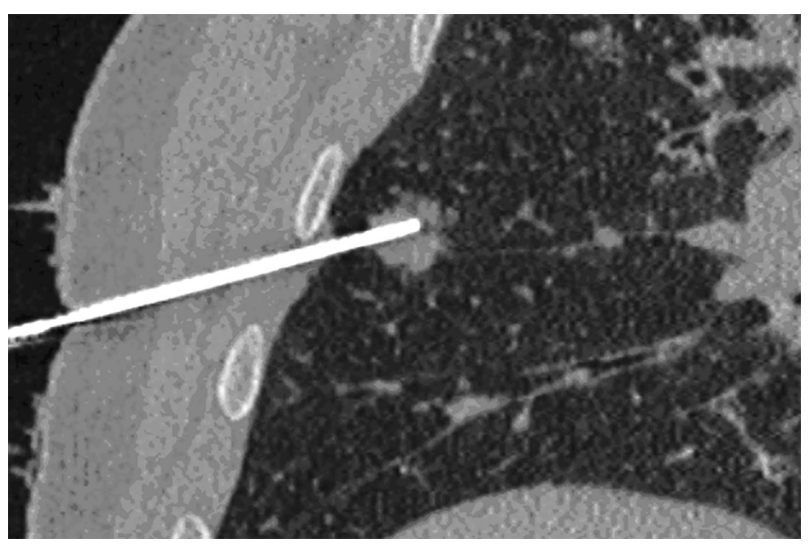

Figure 2 Computed tomography-guided biopsy of a pulmonary nodule in a 62 year-old man. Image reconstruction on a coronal oblique plane showing an I8 gauge needle with the tip inside a solid nodule of the right lung.

Another broad area of extravascular intervention is CTguided tumor ablation. This low-invasive technique allows necrosis of the tumoral mass using different types of energy (eg, radiofrequency, microwave, and laser). CT guidance is indispensable to being able to perform the procedure with precision and safety in order to avoid or minimize damage to surrounding healthy tissues (Figure 3).

CT-guided tumor ablation are mainly performed on lung, liver, kidney, and skeleton; in these cases, the operator cannot monitor the procedure in real time and has to leave the scanning room while acquiring images. The introduction of CT fluoroscopy in the 1990s allowed visualization of the needle in real time and reduced the amount of time required to perform the procedure, partly because there is no need to leave the scanning room. ${ }^{65}$

Percutaneous vertebroplasty and cementoplasty are procedures used to treat fractures in oncology patients with metastasis to bone and in the elderly with osteoporosis. In these cases, a needle is inserted inside the fractured bone in order to inject cement to remodel the damaged structure; CT guidance is used to visualize the position of the needle and to check the distribution of the cement in the bone tissue.

It must be emphasized that performing an image-guided intervention is common practice in most hospitals. CT-guided radiological procedures do expose subjects to radiation, but ease of use and widespread availability make CT a preferred method of guidance, and several methods to minimize radiation exposure are now available. ${ }^{66,67}$

\section{Other emerging and investigative applications of $\mathbf{C T}$}

Currently, several novel techniques and hybrid technologies based on CT have shown promising results. 

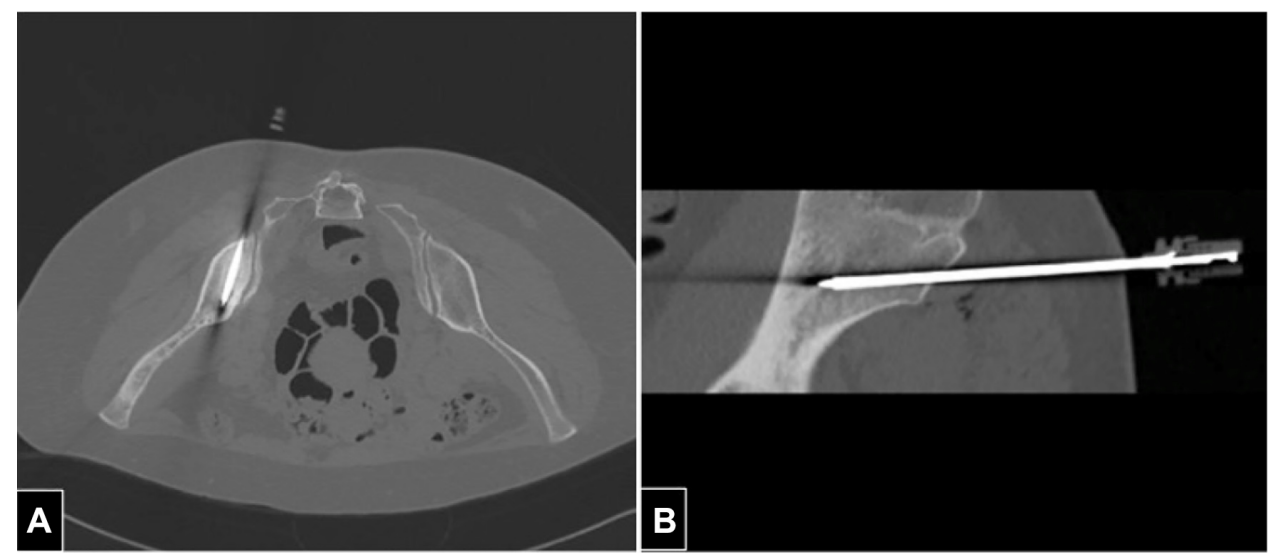

Figure 3 (A and B) Computed tomography-guided microwave ablation of metastasis from breast carcinoma of the left iliac bone in a 54-year-old woman. The patient stands prone and the tip of a 14 gauge needle is inserted into the lesion.

\section{Hybrid PET-CT}

Integration of PET with CT scanners has allowed acquisition of noninvasive three-dimensional images of functional processes occurring in the human body by fusion of images combining anatomy (CT) with function (PET). Since the first prototype was developed in 1990, use of PET/CT in the clinical setting for diagnostic and therapeutic purposes in oncology, neurology, and cardiovascular disease has been growing. Beyer and Pichler reported that hybrid PET/CT has led to a $10 \%-15 \%$ increase in diagnostic accuracy when compared with standalone PET or $\mathrm{CT}^{68}{ }^{6}$ This technique uses CT images for anatomic reference of PET tracer uptake patterns as well as for correction of the PET attenuation data for quantification purposes. ${ }^{69}$ Hybrid PET/CT scanners improve both the detection and treatment of cancer, such as detecting spread of cancer to lymph nodes and assessing the possibility of treatment with radiotherapy. One of the main diagnostic advantages of adding functional data with $\mathrm{CT}$ is the ability to isolate regions of cancer cell metabolism with fluorodeoxyglucose or hypoxic regions using other PET tracers.

\section{X-ray/MR systems}

$\mathrm{X}$-ray/MR systems are hybrid technologies where the combined modality is more than the simple sum of each method. At the same time, they combine the high spatial and temporal resolution of X-ray fluoroscopy, provided by the three-dimensional imaging capabilities, with the soft tissue contrast of MRI. This type of system has become increasingly popular in several diagnostic and minimally invasive applications, eg, for biopsies and arthrograms. ${ }^{70}$ Other potential applications of $\mathrm{X}$-ray/MR systems are: guiding radiation therapy, investigating atherosclerotic plaques, and assessing acute strokes and brain injuries. Two major issues in designing such a system are the bulkiness of the imaging scanners and the conflict in imaging physics, due to rotating metallic parts of X-ray system and magnetic fields of MR one. ${ }^{71}$

\section{Phase-contrast imaging}

Promising results have recently been obtained in material science and biological applications by use of PCI, which can be implemented at third-generation synchrotron radiation sources. ${ }^{72} \mathrm{PCI}$ is an innovative method that is also sensitive to the refraction of X-rays in matter. This technique is based upon the recording attenuation and phase changes of the transmitted X-ray beam and allows evaluation of planar or three-dimensional distribution of the scattering properties of the investigated object. Five techniques have been developed to explore the phase-contrast in the X-ray regime. Of these, the propagation-based phase-contrast imaging method is the simplest way to visualize the phasecontrast. ${ }^{73}$ In the case of biological samples showing weakly absorbing details, use of phase information for imaging is an attractive alternative to conventional X-ray CT. PCI is very appealing because it may help to decrease the total dose absorbed, enhancing the conditions of the entire imaging procedure. Currently, this technique is widely used in preclinical research, but has yet to be tested in pilot clinical trials. ${ }^{74}$ The breast, lung, joints, bone, vasculature, and brain have been mostly imaged in ex vivo and in vitro samples, as well as in a few animal models. Phase-contrast imaging of the breast has been one of the first medical applications of X-ray PCI, reported by Pisano et al. In the musculoskeletal field, PCI allows early and accurate visualization of osteoarthritis and rheumatoid arthritis. ${ }^{75}$ Several groups are exploring the possibility of using PCI in the research and diagnosis of diseases that decrease the alveolar area 
of the lung (eg, emphysema), to investigate lung structure and function, and to detect atelectasis in the injured lung. Some experiments have been performed using PCI on brain tissue for neuroimaging applications, obtaining images of both tumoral and healthy rat brain.

\section{Photon-counting technology for spectral CT}

A new frontier in $\mathrm{CT}$ is photon-counting technology. When an X-ray passes though the body, its spectral distribution changes. However, unlike traditional detectors, photoncounting detectors used in photon-counting spectral CT are able to measure the energy of a single photon. In particular, photon-counting technology is based on selection of a narrow subrange of the spectrum, and measurement of energy attenuation in each window can be used to classify different materials. The challenge of this technology is to record each single photon, with the aim to reduce dose delivered to the patient. Photon-counting detectors are mostly comprised of cadmium telluride, cadmium zinc telluride, and silicon semiconductors. These detectors allow X-ray photons to be counted separately, and cadmium telluride and cadmium zinc telluride detectors also allow measurement of photon energies with a level of accuracy appropriate for clinical application. ${ }^{76}$ Moreover, photon-counting spectral CT overcomes artifact or loss of data due to patient motion, and can achieve good spectral separation between images without requiring heavy prefiltration. Despite some limitations still presented by such detectors (eg, cost of materials, intensity-dependent image artifacts associated with defects in cadmium zinc telluride and cadmium telluride crystals, and limited energy resolution associated with hole trapping and leakage current in cadmium zinc telluride and cadmium telluride materials), many clinical applications are under investigation. ${ }^{77}$

Several studies have investigated the use of photoncounting technology for K-edge imaging. Selective and quantitative imaging of contrast medium can be achieved by exploiting K-edge discontinuity in the photoelectric component of X-ray absorption. An ideal application for $\mathrm{K}$-edge imaging is CT imaging of target-specific and conventional contrast agents that have been designed to be spectral CT-sensitive. ${ }^{78}$

\section{Novel reconstruction algorithms}

The aim of reducing the radiation dose delivered to patient has encouraged the investigation of novel reconstruction algorithms. Unlike traditional back-projection algorithms, iterative reconstruction algorithms use a forward reconstruction model and a precise modeling of scanner geometry and the underlying physics, aiming to produce higher-resolution and low-artifact images than back-projection reconstruction. Among them, the adaptive statistical iterative reconstruction algorithm utilizes information contained in the image reconstructed by back-projections as an initial "building block" in the reconstruction process, to decrease reconstruction time. Model-based iterative reconstruction is demanding in terms of the big number of computers to process data to produce a complete scan, but has the crucial advantage of reducing the radiation dose to $80 \%$ of that for standard reconstruction. ${ }^{79}$

\section{Conclusion}

The introduction of CT is undoubtedly one of the most important milestones achieved in the last 40 years of clinical and biomedical research. The socio-economic impact of CT can be summarized by the installation of more than 50,000 scanners for the year 2010 and by the fact that use of CT in the USA has increased more than three times since 1993 to approximately 70 million scans annually. ${ }^{80}$

In the past, the widespread use of CT was mainly related to its ability to create three-dimensional detailed pictures of areas inside the body and to discriminate soft tissues with good contrast and spatial resolution. These features made the use of CT crucial in many branches of medicine. However, over the years, different solutions have been proposed to minimize the patient dose and to improve image characteristics. For example, since the 1970s, the minimum scan time decreased from about 5 minutes to a fraction of a second, the slice thickness decreased from more than $1 \mathrm{~cm}$ to less than $1 \mathrm{~mm}$, and the resolution improved from $2 \mathrm{pl} / \mathrm{cm}$ to more than $20 \mathrm{pl} / \mathrm{cm}$. The first-generation CTs were time-consuming and were replaced by other solutions, leading rapidly to the fourth-generation CTs. An important milestone in the improvement of CT performance was slip ring technology, which enabled continuous acquisition of data with a reduced scan time. A further revolution was the advancement of MDCT in the late 1990s, which improved temporal and spatial resolution.

The current landscape of CT applications in medicine is growing rapidly, along with new and exciting developments, such as CT-based thermometry and CT-guided invasive diagnostic and therapeutic procedures. Moreover, the introduction of DECT opened up a new perspective regarding the use of CT images. Given that clinical use of spectral CT imaging will be defined by using dual-energy acquisition, progressive utilization of CT data for accurate tissue characterization will 
be possible, gaining significant improvement in oncological and emergency clinical practice. Implementation of DECT technology will decrease the radiation dose exposure for the patient in the daily clinical scenario, which is the main issue of concern with CT scanning at present. Combination of dual-energy acquisition, reduction of X-ray dose, and implementation of techniques for reducing the acquisition time velocity will make $\mathrm{CT}$ imaging more robust and reliable as an evaluation technique for patients in all clinical settings in the future.

\section{Disclosure}

The authors report no conflicts of interest in this work.

\section{References}

1. Cormack AM. Reconstruction of densities from their projections, with applications in radiological physics. Phys Med Biol. 1973;18: 195-207.

2. Hounsfield GN. Computerized transverse axial scanning (tomography) part 1. Description of the system. Br J Radiol. 1973;46:1016-1022.

3. Cierniak R. X-Ray Computed Tomography in Biomedical Engineering. London, UK: Springer-Verlag; 2011.

4. Röntgen WC. Über eine neue Art von Strahlen. Sitzungsberichte der Physikalisch-medizinischen Gesellschaft zu Würzburg [on a new kind of rays]. Science. 1896;3:227-231. German.

5. Thomson E. Stereoscopic roentgen pictures. Electr Eng. 1896;21:256.

6. Bushberg JT. The Essential Physics of Medical Imaging. 2nd ed. Philadelphia, PA, USA: Lippincott Williams and Wilkins; 2002.

7. Cho ZH, Jones JP, Singh M. Foundations of Medical Imaging. 1st ed. New York, NY, USA: John Wiley and Sons; 1993.

8. Kak AC, Slanley M. Principles of Computerized Tomographic Imaging. 1st ed. New York, NY, USA: IEEE Press; 1988.

9. Glover GH. Compton scatter effects in CT reconstructions. Med Phys. 1982;9:860-867.

10. Karçaaltıncaba M, Aktaş A. Dual-energy CT revisited with multidetector CT: review of principles and clinical applications. Diagn Interv Radiol. 2011;17:181-194.

11. Karen MH, Sheila S, Frank C, et al. Multidetector row CT: principles and clinical applications. Crit Rev Comput Tomogr. 2002;43:143-181.

12. Kaza RK, Platt JF, Goodsitt MM, et al. Emerging techniques for dose optimization in abdominal CT. Radiographics. 2014;34:4-17.

13. Cochet H, Dubois R, Sacher F, et al. Cardiac arrythmias: multimodal assessment integrating body surface ECG mapping into cardiac imaging. Radiology. 2014;271:239-247.

14. Godoy MC, Naidich DP, Marchiori E, et al. Basic principles and postprocessing techniques of dual-energy CT: illustrated by selected congenital abnormalities of the thorax. J Thorac Imaging. 2009;24: $152-159$.

15. Aran S, Shaqdan KV, Abujudeh HH. Dual-energy computed tomography (DECT) in emergency radiology: basic principles, techniques, and limitations. Emerg Radiol. 2014;21:391-405.

16. Johnson TRC. Dual-energy CT: general principles. Am J Roentgenol. 2012;199(5 Suppl):3-8.

17. Vogl TJ, Schulz B, Bauer RW, et al. Dual-energy CT applications in head and neck imaging. AJR Am J Roentgenol. 2012;199:S34-S39.

18. Tawfik AM, Kerl JM, Bauer RW, et al. Dual-energy CT of head and neck cancer: average weighting of low- and high-voltage acquisitions to improve lesion delineation and image quality-initial clinical experience. Invest Radiol. 2012;47:306-311.

19. Kang M, Park CM, Lee CH, et al. Dual-energy CT: clinical applications in various pulmonary diseases. Radiographics. 2010;30:685-698.
20. Brown CL, Hartman RP, Dzyubak OP, et al. Dual-energy CT iodine overlay technique for characterization of renal masses as cyst or solid: a phantom feasibility study. Eur Radiol. 2009;19:1289-1295.

21. Graser A, Johnson TR, Hecht EM, et al. Dual-energy CT in patients suspected of having renal masses: can virtual nonenhanced images replace true nonenhanced images? Radiology. 2009;252:433-440.

22. Stolzmann P, Scheffel H, Rentsch K, et al. Dual-energy computed tomography for the differentiation of uric acid stones: ex vivo performance evaluation. Urol Res. 2008;36:133-138.

23. Stolzmann P, Leschka S, Scheffel H, et al. Characterization of urinary stones with dual-energy CT: improved differentiation using a tin filter. Invest Radiol. 2010;45:1-6.

24. Silva AC, Morse BG, Hara AK, Paden RG, Hongo N, Pavlicek W. Dual-energy (spectral) CT: applications in abdominal imaging. Radiographics. 2011;31:1031-1046.

25. Fischer MA, Reiner CS, Raptis D, et al. Quantification of liver iron content with CT-added value of dual-energy. Eur Radiol. 2011;21: 1727-1732.

26. Stolzmann P, Frauenfelder T, Pfammatter T, et al. Endoleaks after endovascular abdominal aortic aneurysm repair: detection with dual-energy dual-source CT. Radiology. 2008;249:682-691.

27. Chandarana H, Godoy MC, Vlahos I, et al. Abdominal aorta: evaluation with dual-source dual-energy multidetector CT after endovascular repair of aneurysms - initial observations. Radiology. 2008;249:692-700.

28. Ruzsics B, Schwarz F, Schoepf UJ, et al. Comparison of dual-energy computed tomography of the heart with single photon emission computed tomography for assessment of coronary artery stenosis and of the myocardial blood supply. Am J Cardiol. 2009;104:318-326.

29. Nagao M, Kido T, Watanabe K, et al. Functional assessment of coronary artery flow using adenosine stress dual-energy CT: a preliminary study. Int J Cardiovasc Imaging. 2011;27:471-481.

30. Schwarz F, Ruzsics B, Schoepf UJ, et al. Dual-energy CT of the heart - principles and protocols. Eur J Radiol. 2008;68:423-433.

31. Zhang LJ, Peng J, Wu SY, Yeh BM, Zhou CS, Lu GM. Dual source dual-energy computed tomography of acute myocardial infarction: correlation with histopathologic findings in a canine model. Invest Radiol. 2010;45:290-297.

32. Bauer RW, Kerl JM, Fischer N, et al. Dual-energy CT for the assessment of chronic myocardial infarction in patients with chronic coronary artery disease: comparison with 3-T MRI. AJR Am J Roentgenol. 2010;195: 639-646.

33. Barreto M, Schoenhagen P, Nair A, et al. Potential of dual-energy computed tomography to characterize atherosclerotic plaque: ex vivo assessment of human coronary arteries in comparison to histology. J Cardiovasc Comput Tomogr. 2008;2:234-242.

34. Boll DT, Merkle EM, Paulson EK, Mirza RA, Fleiter TR. Calcified vascular plaque specimens: assessment with cardiac dual-energy multidetector CT in anthropomorphically moving heart phantom. Radiology. 2008;249:119-126.

35. Hazirolan T, Akpinar B, Unal S, Gümrük F, Haliloglu M, Alibek S. Value of dual energy computed tomography for detection of myocardial iron deposition in thalassaemia patients: initial experience. Eur J Radiol. 2008;68:442-445.

36. Ibrahim E-SH, Bowman AW. Characterization of myocardial iron overload by dual-energy computed tomography compared to T2* MRI. A phantom study. Conf Proc IEEE Eng Med Biol Soc. 2014;2014:5133-5136.

37. Henzler T, Fink C, Schoenberg SO, Schoepf UJ. Dual-energy CT: radiation dose aspects. Am J Roentgenol. 2012;199:S16-S25.

38. Lin XZ, Wu ZY, Tao R, et al. Dual energy spectral CT imaging of insulinoma - value in preoperative diagnosis compared with conventional multi-detector CT. Eur J Radiol. 2012;8:2487-2494.

39. Leschka S, Stolzmann P, Baumüller S, et al. Performance of dual-energy CT with tin filter technology for the discrimination of renal cysts and enhancing masses. Acad Radiol. 2010;17:526-534.

40. Van Werkhoven JM, Gaemperli O, Schuijf JD, et al. Multislice computed tomography coronary angiography for risk stratification in patients with an intermediate pretest likelihood. Heart. 2009;95: 1607-1611. 
41. Hadamitzky M, Freissmuth B, Meyer T, et al. Prognostic value of coronary computed tomographic angiography for prediction of cardiac events in patients with suspected coronary artery disease. JACC Cardiovasc Imaging. 2009;2:404-411.

42. Shuman WP, Branch KR, May JM, et al. Prospective versus retrospective ECG gating for 64-detector CT of the coronary arteries: comparison of image quality and patient radiation dose. Radiology. 2008;248: 431-437.

43. Sommer WH, Albrecht E, Bamberg F, et al. Feasibility and radiation dose of high-pitch acquisition protocols in patients undergoing dualsource cardiac CT. AJR Am J Roentgenol. 2010;195:1306-1312.

44. Einstein AJ, Henzlova MJ, Rajagopalan S. Estimating risk of cancer associated with radiation exposure from 64-slice computed tomography coronary angiography. JAMA. 2007;298:317-323.

45. Einstein AJ, Sanz J, Dellegrottaglie S, et al. Radiation dose and cancer risk estimates in 16-slice computed tomography coronary angiography. J Nucl Cardiol. 2008;15:232-240.

46. Achenbach S, Marwan M, Schepis T, et al. High pitch spiral acquisition: a new mode for coronary angiography. J Cardiovasc Comput Tomogr. 2009;3:117e2.

47. Chinnaiyan KM, Bilolikar AN, Walsh E, et al. CT dose reduction using prospectively triggered or fast-pitch spiral technique employed in cardiothoracic imaging (the CT dose study). $J$ Cardiovasc Comput Tomogr. 2014;8:205-214.

48. Bruder H, Petersilker M, Mehldau H, et al. Flash imaging in dual source CT (DSCT). Proc SPIE 7258, Medical Imaging; 2009. Physics of Medical Imaging, 72580D. Available from: http://proceedings. spiedigitallibrary.org/proceeding.aspx?articleid=1335243. Accessed May 9, 2015.

49. Chinnaiyan KM, Boura JA, DePetris A, et al. Progressive radiation dose reduction from coronary computed tomography angiography in a statewide collaborative quality improvement program: results from the Advanced Cardiovascular Imaging Consortium. Circ Cardiovasc Imaging. 2013;6:646e654.

50. Stafford RJ, Fuentes D, Elliott AA, Weinberg JS, Ahrar K. Laserinduced thermal therapy for tumor ablation. Crit Rev Biomed Eng 2010;38:79-100

51. Lepetit-Coiffé M, Laumonier H, Seror O, et al. Real-time monitoring of radiofrequency ablation of liver tumors using thermal-dose calculation by MR temperature imaging: initial results in nine patients, including follow-up. Eur Radiol. 2010;20:193-201.

52. Saccomandi P, Schena E, Silvestri S. Techniques for temperature monitoring during laser-induced thermotherapy: an overview. Int $J$ Hyperthermia. 2013;29:609-619.

53. Vogl TJ, Straub R, Eichler K, Woitaschek D, Mack MG. Malignant liver tumors treated with MR imaging-guided laser-induced thermotherapy: experience with complications in 899 patients (2,520 lesions). Radiology. 2002;225:367-377.

54. Fani F, Schena E, Saccomandi P, Silcestri S. CT-based thermometry: an overview. Int J Hyperthermia. 2014;30:219-227.

55. Pandeya G, Greuter M, de Jong K, Schmidt B, Flohr T, Outkerk M. Feasibility of noninvasive temperature assessment during radiofrequency liver ablation on computed tomography. J Comput Assist Tomogr. 2011;35:356-360.

56. Li M, Abi-Jaoudeh N, Kapoor A, et al. Towards cone-beam CT thermometry. Proc SPIE. 2013;8671:1-7.

57. Bydder GM, Kreel L. The temperature dependence of computed tomography attenuation values. J Comput Assist Tomogr. 1979;3: 506-510.

58. Fallone BG, Moran PR, Podgorsak EB. Noninvasive thermometry with a clinical X-ray CT scanner. Med Phys. 1982;9:715-721.

59. Mahnken AH, Bruners P. CT thermometry: will it ever become ready for use? Int J Clin Pract. 2011;65 Suppl 171:1-2.
60. Schena E, Saccomandi P, Giurazza F, et al. Monitoring of temperature increase and tissue vaporization during laser interstitial thermotherapy of ex vivo swine liver by computed tomography. Conf Proc IEEE Eng Med Biol Soc. 2013;2013:378-381.

61. Pandeya GD, Klaessens JH, Greuter MJ, et al. Feasibility of computed tomography based thermometry during interstitial laser heating in bovine liver. Eur Radiol. 2011;21:1733-1738.

62. Schena E, Saccomandi P, Giurazza F, et al. Experimental assessment of CT-based thermometry during laser ablation of porcine pancreas. Phys Med Biol. 2013;58:1-12.

63. Alfidi RJ, Haaga J, Meaney TF, et al. Computed tomography of the thorax and abdomen: a preliminary report. Radiology. 1975;117:257-264.

64. Crommelin DJ, Storm G, Luijten P. 'Personalized medicine' through 'personalized medicines': time to integrate advanced, non-invasive imaging approaches and smart drug delivery systems. Int J Pharm. 2011;415:5-8.

65. Katada K, Anno H, Takeshita G, et al. [Development of real-time CT fluoroscopy]. Nippon Igaku Hoshasen Gakkai Zasshi. 1994;54:11721174. Japanese.

66. Sarti M, Brehmer WP, Gay SB. Low-dose techniques in CT-guided interventions. Radiographics. 2012;32:1109-1119.

67. Grasso RF, Cazzato RL, Luppi G, et al. Percutaneous lung biopsies: performance of an optical CT-based navigation system with a low-dose protocol. Eur Radiol. 2013;23:3071-3076.

68. Beyer T, Pichler B. A decade of combined imaging: from a PET attached to a CT to a PET inside an MR. Eur J Nucl Med Mol Imaging. 2009;36:S1-S2

69. Kinahan P, Townsend D, Beyer T, Sashin D. Attenuation correction for a combined 3D PET/CT scanner. Med Phys. 1998;25:2046-2053.

70. Pelc NJ. Hybrid $x$-ray/MR system and other hybrid imaging modalities. In: Medical Imaging 2003: Physiology and Function: Methods, Systems, and Applications. Clough AV; Amini AA, editors. Proceedings of SPIE; San Diego, CA. Volume 5031 February 15; 2003.

71. Ganguly A, Wen Z, Daniel B, et al. Truly hybrid X-ray/MR imaging: towards a streamlined clinical system. Acad Radiol. 2005;12: 1167-1177.

72. Baik S, Kim HS, Jeong MH, et al. International consortium on phase contrast imaging and radiology beamline at the Pohang light source. Rev Sci Instrum. 2004;75:4355-4358.

73. Snigirev A, Snigireva I, Kohn V, et al. On the possibilities of x-ray phase contrast microimaging by coherent high-energy synchrotron radiation. Rev Sci Instrum. 1995;66:5486-5492.

74. Castelli E, Tonutti M, Arfelli F, et al. Mammography with synchrotron radiation: first clinical experience with phase-detection technique. Radiology. 2011;259:684-694.

75. Pisano ED, Johnston RE, Chapman D, et al. Human breast cancer specimens: diffraction-enhanced imaging with histologic correlationimproved conspicuity of lesion detail compared with digital radiography. Radiology. 2000;214:895-901.

76. Shikhaliev PM, Shannon GF. Photon counting spectral CT versus conventional CT: comparative evaluation for breast imaging application. Phys Med Biol. 2011;56:1905-1930.

77. Feuerlein S, Roessl E, Proksa R, et al. Multienergy photon-counting K-edge imaging: potential for improved luminal depiction in vascular imaging. Radiology. 2008;249:1010-1016.

78. Schlomka J, Roessl E, Dorscheid R, et al. Experimental feasibility of multi-energy photon-counting K-edge imaging in pre-clinical computed tomography. Phys Med Biol. 2008;53:4031-4037.

79. Singh S, Kalra MK, Hsieh J, et al. Abdominal CT: comparison of adaptive statistical iterative and filtered back projection reconstruction techniques. Radiology. 2010;257:373-383.

80. Berrington de González A, Mahesh M, Kim KP, et al. Projected cancer risks from computed tomographic scans performed in the United States in 2007. Arch Intern Med. 2009;169:2071-2077. 
Medical Devices: Evidence and Research

Dovepress

\section{Publish your work in this journal}

Medical Devices: Evidence and Research is an international, peerreviewed, open access journal that focuses on the evidence, technology, research, and expert opinion supporting the use and application of medical devices in the diagnosis, treatment and management of clinical conditions and physiological processes. The identification of novel

devices and optimal use of existing devices which will lead to improved clinical outcomes and more effective patient management and safety is a key feature. The manuscript management system is completely online and includes a quick and fair peer-review system. Visit http://www. dovepress.com/testimonials.php to read real quotes from authors.

Submit your manuscript here: http://www.dovepress.com/medical-devices-evidence-and-research-journal 